\title{
Erratum to: CT-Guided Percutaneous Trans-scapular Lung Biopsy in the Diagnosis of Peripheral Pulmonary Lesion Nodules of the Superior Lobes Using Large Needles
}

\author{
Alberto Rebonato $^{1}$ - Daniele Maiettini ${ }^{1}$ - Marco Andolfi ${ }^{2}$ - Matthias J. Fischer ${ }^{3}$. \\ Jacopo Vannucci ${ }^{2} \cdot$ Giulio Metro $^{4}$ - Antonio Basile ${ }^{5} \cdot$ Michele Duranti $^{3}$. \\ Michele Rossi ${ }^{6}$
}

(c) Springer Science+Business Media, LLC and the Cardiovascular and Interventional Radiological Society of Europe (CIRSE) 2017

\section{Erratum to: Cardiovasc Intervent Radiol DOI 10.1007/s00270-017-1768-0}

In the published article, Michele Rossi mistakenly appears as the next to the last name in the author list. Michele Rossi should appear as the last name in the author list as shown in this erratum.
The online version of the original article can be found under doi: $10.1007 / \mathrm{s} 00270-017-1768-0$.
Alberto Rebonato
rebonatoalberto@gmail.com
Daniele Maiettini
danielemaiettini@gmail.com
Marco Andolfi
marcoandolfi@hotmail.com
Matthias J. Fischer
mfischer@alice.it
Jacopo Vannucci
jacopovannucci@tiscali.it
Giulio Metro
giulio.metro@yahoo.com
Antonio Basile
antodoc@yahoo.com
Michele Duranti
micheleduranti@aopg.it
Michele Rossi
michele.rossi@uniroma1.it

Radiology Unit, Department of Surgical and Biomedical Science, Santa Maria della Misericordia University Hospital, Perugia University, Sant'Andrea delle Fratte, 06132 Perugia, Italy

2 Department of Thoracic Surgery, S. Maria della Misericordia Hospital, University of Perugia Medical School, Sant'Andrea delle Fratte, 06132 Perugia, Italy

3 Department of Radiology, S. Maria della Misericordia Hospital, University of Perugia Medical School, Sant'Andrea delle Fratte, 06132 Perugia, Italy

4 Department of Medical Oncology, S. Maria della Misericordia Hospital, University of Perugia Medical School, Sant'Andrea delle Fratte, 06132 Perugia, Italy

5 Department of Diagnostic and Interventional Radiology, Ospedale Ferrarotto, Via Trieste 14, 95127 Catania, Italy

6 Department of Radiology, Sant'Andrea Hospital, Faculty of Medicine and Psychology, Sapienza University, Rome, Italy 


\title{
CT-Guided Percutaneous Trans-scapular Lung Biopsy in the Diagnosis of Peripheral Pulmonary Lesion Nodules of the Superior Lobes Using Large Needles
}

\author{
Alberto Rebonato $^{1}$ - Daniele Maiettini ${ }^{1}$ - Marco Andolfi ${ }^{2}$ - Matthias J. Fischer ${ }^{3}$. \\ Jacopo Vannucci $^{2}$ - Giulio Metro ${ }^{4}$ Antonio Basile ${ }^{5}$ Michele Rossi $^{6}$. \\ Michele Duranti ${ }^{3}$
}

Received: 18 April 2017/ Accepted: 9 August 2017

(C) Springer Science+Business Media, LLC and the Cardiovascular and Interventional Radiological Society of Europe (CIRSE) 2017

\begin{abstract}
Purpose CT-guided percutaneous transthoracic lung needle biopsy (PLB) is an effective procedure for obtaining cyto-histological diagnoses of peripheral lesions. However, upper postero-lateral lung nodules (UPLN) may be difficult to reach using a standard intercostal either anterior or lateral approach or when the nodule is just behind a rib or scapula. We evaluated the feasibility and effectiveness of trans-scapular approach (tPLB) in these patients using large-core needles.

Methods We retrospectively collected the data of 11 consecutive patients (mean age 74.6 years, SD 5.9) among those scheduled to undergo CT-guided PLB over the period February 2015 to February 2017. In these patients, the presence of a UPLN required a tPLB using a co-axial
\end{abstract}

Alberto Rebonato

rebonatoalberto@gmail.com

Daniele Maiettini

danielemaiettini@gmail.com

Marco Andolfi

marcoandolfi@hotmail.com

Matthias J. Fischer

mfischer@alice.it

Jacopo Vannucci

jacopovannucci@tiscali.it

Giulio Metro

giulio.metro@yahoo.com

Antonio Basile

antodoc@yahoo.com

Michele Rossi

michele.rossi@uniroma1.it

Michele Duranti

micheleduranti@aopg.it technique and large full-core needles (15G for the scapular piercing and 18-19G for tissue sampling). All patients were assessed by telephone at $24 \mathrm{~h}, 78 \mathrm{~h}$ and at an office visit at 30 days after the procedure to evaluate post-procedural pain (VAS score) and shoulder mobility.

Results Ten of the eleven samples were diagnostic. No major complications were encountered. Three patients developed a pneumothorax, but none required pleural drainage. All the patients confirmed the absence of pain at 24-72 $\mathrm{h}$ and 30 days, reporting a VAS score less than 1, without any shoulder mobility limitation.

Conclusion tPLB seems to be an effective and feasible procedure, accompanied by a low risk of pneumothorax in UPLN cases.

Radiology Unit, Department of Surgical and Biomedical Science, Santa Maria della Misericordia University Hospital, Perugia University, Sant'Andrea delle Fratte, 06132 Perugia, Italy

2 Department of Thoracic Surgery, S. Maria della Misericordia Hospital, University of Perugia Medical School, Sant'Andrea delle Fratte, 06132 Perugia, Italy

3 Department of Radiology, S. Maria della Misericordia Hospital, University of Perugia Medical School, Sant'Andrea delle Fratte, 06132 Perugia, Italy

4 Department of Medical Oncology, S. Maria della Misericordia Hospital, University of Perugia Medical School, Sant'Andrea delle Fratte, 06132 Perugia, Italy

5 Department of Diagnostic and Interventional Radiology, Ospedale Ferrarotto, Via Trieste 14, 95127 Catania, Italy

6 Department of Radiology, Sant'Andrea Hospital, Faculty of Medicine and Psychology, Sapienza University, Rome, Italy 
Keywords Biopsy needle · Image-guided biopsy · Lung neoplasms $\cdot$ Trans-scapular

\section{Introduction}

Lung cancer is the leading cause of cancer-related mortality worldwide with an overall 5-year survival rate of 17\% [1]. Tissue biopsy remains the gold standard for diagnosing both malignant and non-malignant diseases and for obtaining important information for targeted therapies. Although flexible bronchoscopy and other endoscopic techniques (e.g., trans-bronchial needle aspiration TBNA, endo-bronchial ultrasounds EBUS) are the recommended procedures for patients suspected of having lung cancer $[1,2]$, surgical or percutaneous tissue biopsy is required in some cases.

Many studies have investigated the precautions that should be considered for reducing complications while performing percutaneous lung biopsies (PLB) [3-5]. Pneumothorax (PTX) remains the most prevalent complication of PLB, with a reported incidence rate that ranges from 8 to $65 \%$ [2-4]. Predicting PTX is still difficult: the nodule depth (pleura-lesion distance), small lesion's size, repeated pleural punctures, crossed fissures and emphysema are considered relevant risk factors for PTX associated with CT-guided PLB [3]. For these reasons, some guidelines are recommended for performing PLB, such as avoiding the fissures and blebs or choosing the least pleuralesion distance $[5,6]$. However, certain pulmonary nodules may be difficult to reach using the conventional approach, due to their location behind osseous structures, even though some authors have suggested trans-osseous routes [7, 8]. The previously described trans-scapular technique provides for the use of small needles (22G). According to the literature on lung biopsies, small needles are susceptible to similar complication rates as large needles (18-19G) [3].

We retrospectively collected the data for all patients who had undergone CT-guided full-core PLB with a transscapular approach technique (tPLB) of upper postero-lateral lung nodules (UPLN) in order to assess the safety and feasibility of the technique using large-core needles.

\section{Materials and Methods}

\section{Study Design and Population}

This retrospective non-comparative study was conducted in a tertiary care hospital (about 300 CT-guided PLB per year). Among patients who underwent CT-guided PLB over the period February 2015-2017, we collected data for all the cases in which a tPLB was performed (6 male, 5 female; mean age 74.6 years, SD 5.9). All patients gave informed written consent prior to the procedure and waived institutional review board (IRB) approval.

The pre-procedure evaluation included detailed history, physical examination, blood exams and cardiopulmonary assessment. All cases requiring PLB are discussed in the multidisciplinary meeting. The bronchoscopist, IR and surgeons discussed the best diagnostic option for obtaining cytologic/histologic samples, in consultation with the pathologist.

A bronchoscopy was unsuccessfully attempted in 4 cases, however, it was considered unfeasible in the remaining 6 cases. A patient's eligibility criteria for biopsy are: platelet count $>50,000 / \mathrm{mm}^{3}$, INR $<1.5$, and must have been deemed a poor candidate for a previous nondiagnostic trans-bronchial biopsy. The performing physician evaluated, on pre-biopsy and preliminary images, all the variables considered to be risk factors that could lead to complications: crossing fissures, crossing blebs, angulation of pleural puncture, vascular structures, nodule depth etc. The decision to perform a tPLB was taken intra-procedurally, in some cases after the failure of conventional approaches (Fig. 1).

\section{CT-Guided TNAB with Trans-scapular Approach Technique}

All procedures used in this study were performed by two interventional radiologists with 10 and 30 years of experience in IR. The patient was positioned prone or lying in the lateral decubitus on the opposite side of nodule localization (Fig. 1A), after having been asked to find the most comfortable position, without conscious sedation. The patient was instructed to breathe regularly, avoiding any movement, and he was not required to hold his breath. After preliminary image analysis, the entry point was marked on the patient's skin using a dermographic pen and gantry's laser pointer. Larger scapular arteries were located on the margins of the scapular body, so peripheral entry points on the scapula were avoided [9].

Local anesthesia (2\% lidocaine) was performed with a $22 \mathrm{G}$ needle from the skin to the scapular cortex (Fig. 1B, C). The periostium of the scapula was accurately infiltrated. Once the biopsy pathway was defined, a small incision was made to the skin, and a $15 \mathrm{G}$ bone marrow biopsy needle "ERG" type (Selective "ERG" type, ZAMAR d.o.o., Porec, Croatia) was inserted in the scapular body using a drilling and steady pressure movement (Fig. 1D) or using a surgical hammer. Then, an $18 \mathrm{G}$ (Biomol, HS Hospital Service S.p.A., Aprilia, Italy) or 19G (Hepashot, Vigeo SrL, Bagnolo San Vito, Italy) Menghini- 


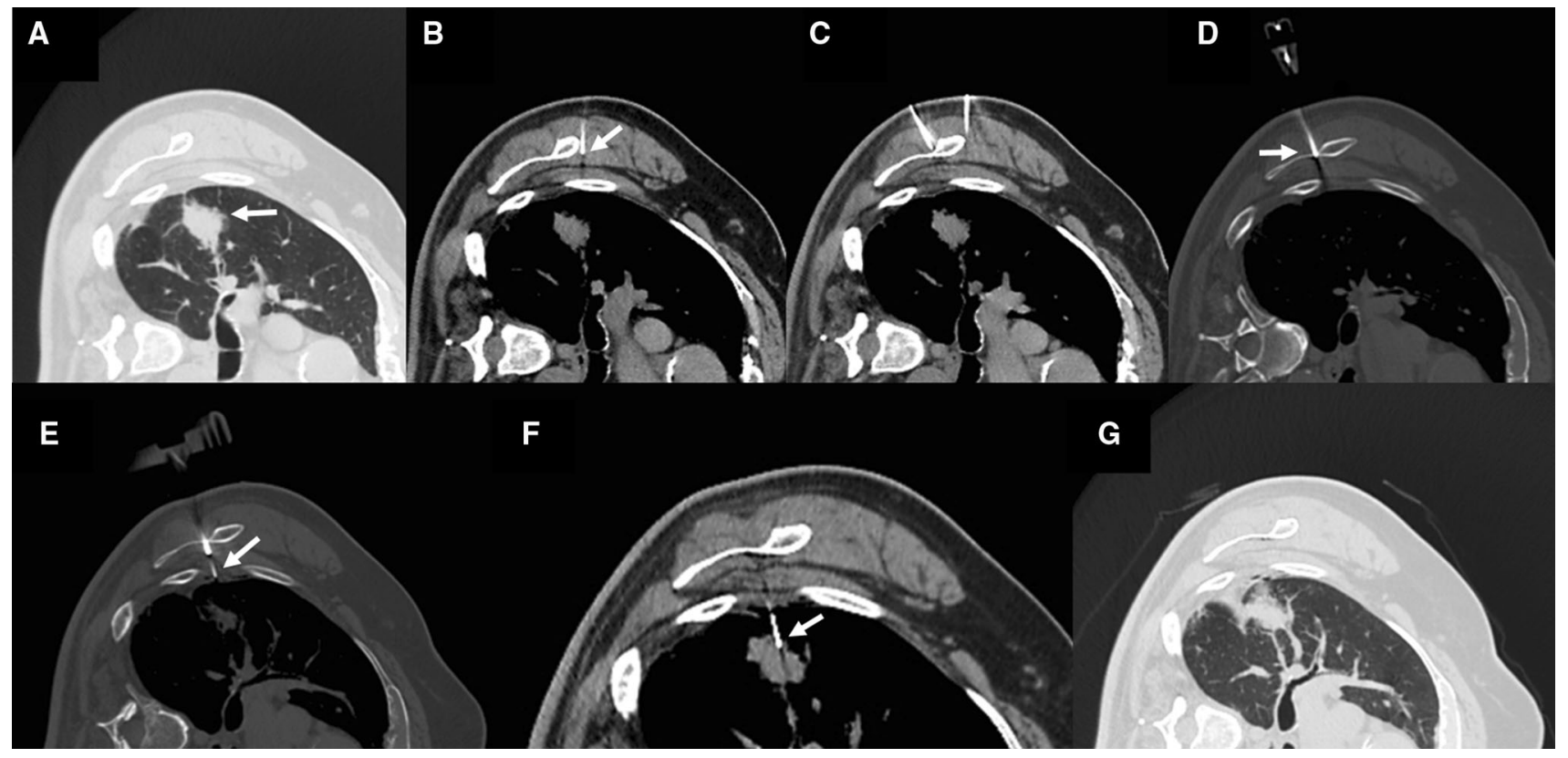

Fig. 1 A Patient presenting a 33-mm nodular lesion of the right superior lobe (arrow), pleura-lesion distance was $15 \mathrm{~mm}$. Patient was positioned lying on the left side. B A first attempt of PLB was made passing between the anterior margin of the scapula and a rib, note the $22 \mathrm{G}$ needle for anesthesia (arrow). Even moving the patient arm did not allow a constant open needle track. C Consequently, we decided to perform a trans-scapular access. Note the second $22 \mathrm{G}$ needle used for local anesthesia near the scapular body. D A $15 \mathrm{G}$ bone marrow biopsy needle Selective "ERG" type was inserted in the scapular

type semi-automatic full-core biopsy needle (with a two cm cutting length) was coaxially inserted and gently advanced to the pleura (Fig. 1E) without a guiding needle. More lidocaine was injected through the Menghini needle just over the pleura to obtain a small meniscus for a complete anesthesia. Then, the needle was advanced into the lesion to collect the sample using a single movement (Fig. 1F). A CT scan was performed after the removal of all needles (Fig. 1G). In all cases, only one sample was obtained and was placed in a $4 \%$ formaldehyde solution (BiopSafe, Axlab A/S, Vedbaek, Denmark).

The procedure was performed in a day-hospital setting. The patients were kept under observation for $6 \mathrm{~h}$, and a chest X-ray was performed $3 \mathrm{~h}$ after the biopsy. Vital signs and referred symptoms were assessed prior to discharge. Asymptomatic PTXs and/or small PTXs $(<2 \mathrm{~cm}$ between the lung margin and the chest wall at the level of the hilum on the chest X-ray) were treated conservatively. A chest tube was inserted for evacuation in patients with PTXs $>2 \mathrm{~cm}$ and/or with signs of shortness of breath or respiratory distress $[10,11]$. Significant muscle and skeletal bleeding were assessed during and at the end of the procedure using the CT scans since such complications could occur soon after or during the needle placement. Late bleeding was ruled out by the complete absence of body (arrow). E Then, an 18G Menghini-type semi-automatic fullcore biopsy needle was coaxially inserted and gently advanced to the parietal pleura (arrow), where more local anesthesia was injected. F The Menghini needle was firmly advanced in one movement into the lesion (arrow). A CT scan confirmed the needle placement in the lesion, and then the sample was gathered. G Post-procedure CT scan performed after the removal of all needles showed the absence of PTX, note small air bubbles on the external side of parietal pleura, site of anesthesia

discomfort/pain or limitation of movement at the site of the puncture.

\section{Data Analysis}

Data were collected on a standardized form developed for this purpose. In addition to the identifying and demographics data, the following procedure details were collected: scapular thickness, scapular and pleural depth from the skin, nodule axial maximum diameter, distance from the pleura, number of scans performed and radiation dose. The histological results were collected along with the presence of complications: pneumothorax, pulmonary hemorrhage, hemoptysis, hemothorax, air embolism and death. All patients were assessed by telephone at $24 \mathrm{~h}, 78 \mathrm{~h}$ and during an office visit at 30 days following the procedure to evaluate post-procedural pain (VAS score) and shoulder mobility.

\section{Results}

The locations of pulmonary lesions were found in the posterior segment of the right upper lobe in 7 cases, in the apico-posterior segment of the left upper lobe in 4 cases 
and in the apical segment of the left inferior lobe in one case. The mean scapular thickness, lesion's axial maximum diameter, scapular and pleural depth from the skin were, respectively, 2.0, 28.2, 30 and $50 \mathrm{~mm}$ (ranges 1.4-2; 20-3; 26-3; 45-57 mm, respectively). In 4 cases, the lesion was adherent to the pleura, while in the remaining 7 cases the mean distance between the pleura and the lesion was $6.9 \mathrm{~mm}$ (range $4-15 \mathrm{~mm}$ ).

The histology of such pulmonary nodules was of adenocarcinoma in eight cases. In one of them, the sample was insufficient for diagnosis (adenocarcinoma was diagnosed on the surgical specimen). The remaining three patients had a squamous cell carcinoma. All the samples allowed for gene mutational analysis except for the one with insufficient material for diagnosis.

Two patients showed a 1- and 4-mm PTX layer on the CT scan performed after the needle removal. At the 3-h thoracic X-ray, the pneumothorax disappeared in one patient and increased in the other one (from 1 to $25 \mathrm{~mm}$ ), while a third patient developed a 13-mm pneumothorax. None of the patients required a pleural evacuation. Data are summarized in Table 1. Only one case of pulmonary hemorrhage was present on the post-procedure CT. No cases of either minor or major complications were encountered [12]. All the patients confirmed the absence of pain at 24-72 $\mathrm{h}$ and 30 days, reporting a VAS score less than 1 , without any shoulder mobility limitation.

\section{Discussion}

The overall amount of PLB has increased in recent years due to the evolution of clinical oncology and its related new demands [13, 14]. The detection of lung nodules during screening [15] or unrelated reasons has increased with the diffusion of CT scanners. Moreover, the development of targeted therapies has led to an increasing demand for molecular tissue characterization and re-characterization [16] which has enabled practitioners to direct patients to the most appropriate treatment option according to tumor bioprofile [13, 14, 17].

A UPLN could be a challenging target for a biopsy. The anterior approach increases the pleura-lesion distance with a higher risk of PTX due to the length of traversed parenchyma [18]. As a result, the subclavian neurovascular bundle must be avoided. The major fissure often lies in the biopsy needle path if target lesions are located in the superior segment of the lower lobe [3-5]. In some cases, a very angulated needle insertion, tangentially to the pleural surface, may be repeatedly required in cases of unsuccessful tissue or cytologic collection [6]. Therefore, the posterior approach with the patient in prone position is often preferred, particularly for posterior lesions. However, in select patients the presence of ribs and scapula can be a technical obstacle for the subpleural UPLNs. Ribs, and similarly the edge of the scapula, can usually be avoided by angulating the needle. In such cases, the angulation of CT gantry may be of help to the operator. TBNA and other bronchoscopic techniques revealed good results in lung tumor sampling. Even so, peripheral or subpleurical lesions could be challenging for these techniques. The UPLNs considered in this study were adherent to the pleura in 4 cases and at an average distance of $7 \mathrm{~mm}$ from the pleura in the remaining cases. Such sites could often be difficult using bronchoscopy [2].

If the nodules are underneath the scapular body, a direct nodule biopsy could be obtained with trans-scapular access; an average of 300 PLB are performed at our institution, and 11 tPLB in 2 years represent about the $2 \%$ of the procedures. In effect, the low incidence hampers rapid learning curves. Regardless, the use of co-axial technique should be part of the interventional radiologists' regular daily practice.

In this series, the trans-scapular approach reduced this pleura-lesion distance to zero in 5 cases, since the nodule had a pleural contact, and to a mean of $7 \mathrm{~mm}$ in the remaining cases. Moreover, in the case of severe emphysema, the presence of bullae, surrounding a subpleural lesion, could decrease the spectrum of options for safe direct access. In fact, a trans-scapular approach could be the only way to obtain a safe route. The pneumothorax rate is about $25 \%$ in a large meta-analysis accompanied by $5 \%$ requiring intervention [3]. The present study PTX rate overlaps these data $(27 \%-3 / 11)$; however, no intervention was required. Moreover, there was no case of hemoptysis and just one case $(9 \%)$ of pulmonary hemorrhage, showing a reduction in such complications, which represent, respectively, about 4 and $18 \%$ of cases [3]. In all these patients, the complication observed was asymptomatic. So, these patients were discharged in the late afternoon with the warning to advise the hospital staff or to come back to the institution if any respiratory or correlated symptom occurred (e.g., hemoptysis or dyspnea) (Fig. 2).

Rossi et al performed the trans-scapular approach in five patients using a co-axial technique penetrating the scapular bone with an 18G two-piece guide needle [8]. Following this, a $22 \mathrm{G}$ biopsy needle was used for cytological sample collection. In our experience, we opted for larger needles, both for trans-scapular piercing and sample collection. Recent studies have demonstrated that both fine-needle and core biopsy have a similar complication rate [3, 19]. With regard to the possibility of tumor seeding, since the technique provides the placement of a trans-bone cannula up to the pleural surface, the needle that crosses the tumor does not actually cross the thoracic wall. As a result, we cannot 
A. Rebonato et al.: CT-Guided Percutaneous Trans-scapular Lung Biopsy in the Diagnosis of...

Table 1 Population and procedure characteristics

\begin{tabular}{|c|c|c|c|c|c|c|c|c|c|c|}
\hline Patient & Sex & Age & Side & $\begin{array}{l}\text { Lesion } \\
\text { diameter }\end{array}$ & $\begin{array}{l}\text { Skin-pleura } \\
\text { distance }(\mathrm{mm})\end{array}$ & $\begin{array}{l}\text { Skin-scapula } \\
\text { distance }(\mathrm{mm})\end{array}$ & $\begin{array}{l}\text { Pleura-nodule } \\
\text { distance }(\mathrm{mm})\end{array}$ & $\begin{array}{l}\text { Scapular } \\
\text { thickness } \\
(\mathrm{mm})\end{array}$ & $\begin{array}{l}\text { Periprocedural } \\
\text { complications } \\
\text { (PTX in mm) }\end{array}$ & $\begin{array}{l}3 \text { h chest X-ray } \\
(\text { PTX in } \mathrm{mm} \text { ) }\end{array}$ \\
\hline 1 & $\mathrm{~F}$ & 80 & $\mathrm{R}$ & 25 & 49 & 31 & 0 & 1.4 & 0 & 0 \\
\hline 2 & M & 78 & $\mathrm{R}$ & 33 & 57 & 31 & 15 & 2 & 0 & 13 \\
\hline 3 & M & 71 & $\mathrm{R}$ & 33 & 50 & 28 & 0 & 2.5 & 1 & 25 \\
\hline 4 & $\mathrm{~F}$ & 74 & $\mathrm{~L}$ & 25 & 46 & 30 & 0 & 1.8 & 0 & 0 \\
\hline 5 & M & 69 & $\mathrm{R}$ & 34 & 48 & 26 & 6 & 2.1 & 0 & 0 \\
\hline 6 & M & 81 & $\mathrm{R}$ & 22 & 53 & 29 & 8 & 2 & 0 & 0 \\
\hline 7 & $\mathrm{~F}$ & 67 & $\mathrm{~L}$ & 20 & 47 & 32 & 0 & 2 & 0 & 0 \\
\hline 8 & $\mathrm{~F}$ & 78 & $\mathrm{~L}$ & 35 & 55 & 28 & 5 & 1.7 & 0 & 0 \\
\hline 9 & M & 82 & $\mathrm{~L}$ & 29 & 52 & 33 & 4 & 2.3 & 0 & 0 \\
\hline 10 & M & 65 & $\mathrm{~L}$ & 28 & 45 & 35 & 6 & 1.5 & 4 & 0 \\
\hline 11 & $\mathrm{~F}$ & 76 & $\mathrm{R}$ & 26 & 48 & 27 & 4 & 2.2 & 0 & 0 \\
\hline Mean & & 74.6 & & 28.2 & 50.0 & 30.0 & 4.4 & 2.0 & 0.5 & 3.5 \\
\hline SD & & 5.9 & & 5.1 & 3.8 & 2.7 & 4.6 & 0.3 & 1.2 & 8.1 \\
\hline Max & & 82.0 & & 35.0 & 57.0 & 35.0 & 15.0 & 2.5 & 4.0 & 25.0 \\
\hline Min & & 65.0 & & 20.0 & 45.0 & 26.0 & 0.0 & 1.4 & 0.0 & 0.0 \\
\hline
\end{tabular}

$P T X$ pneumothorax; SD standard deviation; $L$ left; $R$ right

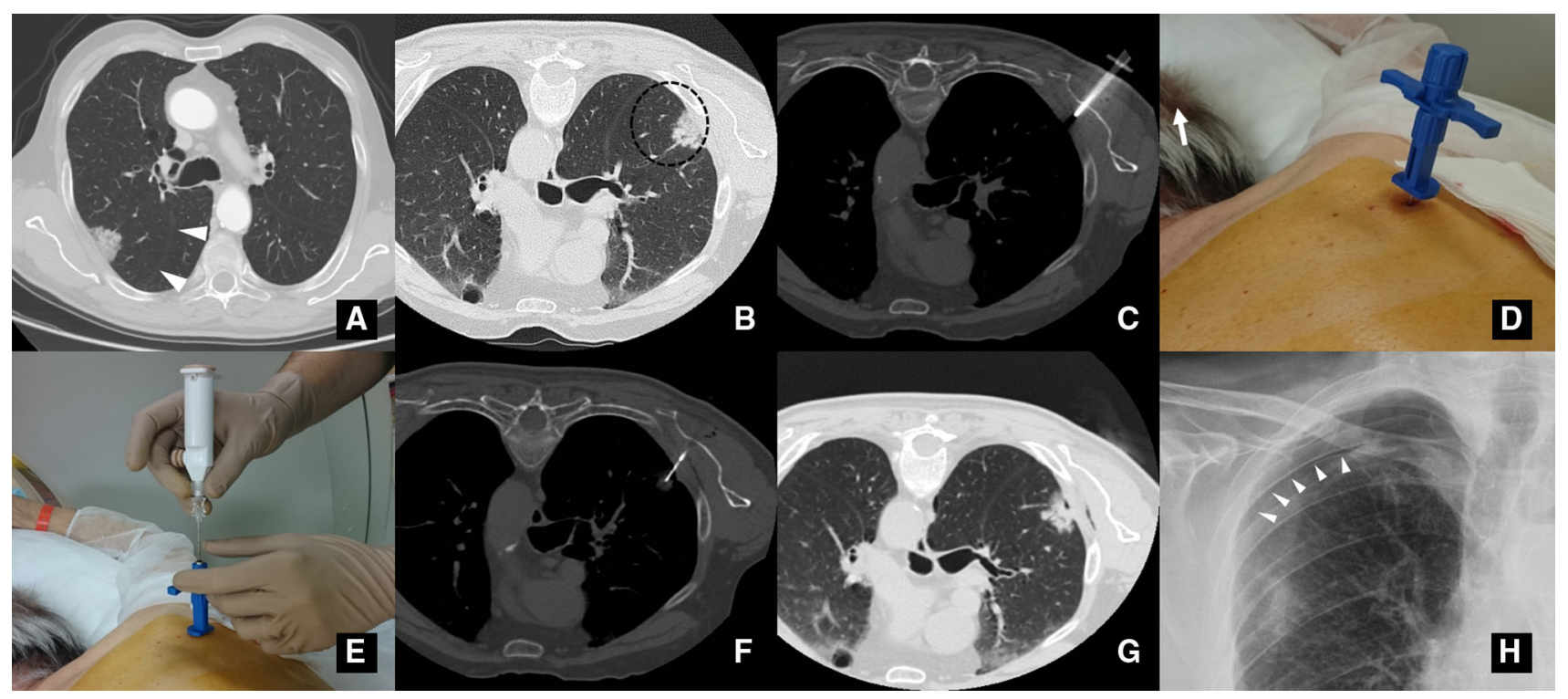

Fig. 2 A Lung contrast-enhanced CT scan performed on a patient presenting a 33-mm nodular lesion of the right superior lobe in contact with the pleura, 40 days prior to the biopsy. Note the long path required supposing an anterior approach, a lateral approach required an acute angle piercing the pleura, while the posterior approach was hampered by the fissure (white arrow heads). B Patient was positioned prone the day of the procedure, the position of the fissure and of the ribs was confirmed, and the trans-scapular approach was preferred. C, D A $15 \mathrm{G}$ bone marrow biopsy needle Selective "ERG" type was inserted in the scapular body. E Then, a 19G Menghini-type semi-automatic full-core biopsy needle was coaxially inserted and gently advanced to the parietal pleura. The Menghini needle was firmly advanced in one movement in the lesion (arrow). F A CT scan confirmed the needle placement in the lesion. G Postprocedure CT scan performed after the removal of all needles shows the absence of PTX. Note small air bubbles on the external side of parietal pleura and intercostal muscles, site of anesthesia. H Postprocedure $3 \mathrm{~h}$ chest $\mathrm{X}$-ray in expiratory projection showing a delayed development of a 25-mm PTX (arrow heads), but pleural drainage was not required. The patient was discharged in the late afternoon with the warning to advise the hospital staff or to come back to the institution if any respiratory or correlated symptom occurred 
expect a significant increased risk of seeding as compared to the standard technique.

Due to the increase in requests for molecular profiling of lung cancer $[13,14,16]$, we prefer the use of $18 \mathrm{G}$ core needles to obtain a larger amount of tissue. Non-surgical histologic biopsies are considered to have a higher diagnostic yield, although some authors reported a comparable diagnostic accuracy for needle aspiration [19]. However, there are no studies available comparing the diagnostic yield of fine-needle or core biopsy in the diagnosis of molecular predictive markers, nor quantifying the amount of tumoral tissue obtained [20]. Consequently, more evidence is necessary and further focused research should be encouraged.

The use of two needles for performing the biopsy could raise some concerns about the cost and timing of the procedure. Considering the low rate of nodules requiring this approach, the overall cost can easily be absorbed into the biopsies performed per year. Moreover, the risk of insufficient sampling, accompanied by the necessity to repeat the procedure, or the cost of complication management could reasonably justify the relatively low cost addition to the procedure. The procedure time does not seem to be longer than the time usually required for difficult nodule localizations.

Considering the mean scapular thickness of $2 \mathrm{~mm}$ reported in our study, the use of a $15 \mathrm{G}$ bone marrow biopsy needle ensures easier penetration; however, notwithstanding, we had to resort to using a surgical hammer twice to succeed in scapular penetration. Moreover, the $15 \mathrm{G}$ needle ensures some degrees of steering during the co-axial introduction of the $18 \mathrm{G}$ one. This could be useful considering the inability to steer the first needle once inserted in the scapula. According to the other available series [7, 8], none of the patients reported bleeding or other complications after the scapula penetration, even with the use of a $15 \mathrm{G}$ needle.

This technique seemed to be well tolerated if an optimal anesthetic infiltration of the anterior and posterior periostium of the scapular body and of the pleura was performed. This appears to be a key measure when performing the procedure and avoids the need for conscious sedation or intravenous analgesia. Local anesthesia can be easily achieved on the posterior surface of the scapular body with the $22 \mathrm{G}$ needle. The anterior surface can be anesthetized through the $15 \mathrm{G}$ needle just after anterior cortex piercing, without causing significant discomfort to the patient. Also during the follow-up, all patients experienced an absence of pain associated with unchanged shoulder mobility.

The small number of patients enrolled limits this. In our experience, enrolling a large series of patients in order to set up prospective, randomized studies that compare the trans-scapular approach to the conventional PLB is a difficult task due to the limited need for trans-scapular access in our institution. Even so, further studies are needed to confirm these preliminary data. The lesions in this paper had a relatively large mean diameter $(28 \mathrm{~mm})$, which could influence the high reported diagnostic rate, but still allows a valid evaluation of its feasibility.

In summary, this study supports the feasibility and safety of the tPLB using full-core biopsy needles, accompanied by a low risk of PTX and a promising diagnostic accuracy. Excessively challenging conventional approaches to UPLN could be converted to trans-scapular approach with minor concerns.

\section{Compliance with Ethical Standards}

Conflict of interest The authors declare that they have no conflict of interests.

Informed Consent Informed consent was obtained from all individual participants included in the study.

\section{References}

1. Rivera MP, Mehta AC, Wahidi MM. Establishing the diagnosis of lung cancer: diagnosis and management of lung cancer, 3rd ed: American College of Chest Physicians evidence-based clinical practice guidelines. Chest. 2013;143(5 Suppl):e142S-65S.

2. Andolfi M, Potenza R, Capozzi R, Liparulo V, Puma F, Yasufuku $\mathrm{K}$. The role of bronchoscopy in the diagnosis of early lung cancer: a review. J Thorac Dis. 2016;8(11):3329-37.

3. Heerink WJ, de Bock GH, de Jonge GJ, Groen HJ, Vliegenthart R, Oudkerk M. Complication rates of CT-guided transthoracic lung biopsy: meta-analysis. Eur Radiol. 2017;27(1):138-48.

4. Klein JS, Zarka MA. Transthoracic needle biopsy. Radiol Clin N Am. 2000;38(2):235-66.

5. Yankelevitz DF, Vazquez M, Henschke CI. Special techniques in transthoracic needle biopsy of pulmonary nodules. Radiol Clin N Am. 2000;38(2):267-79.

6. Ko JP, Shepard JO, Drucker EA, Aquino SL, Sharma A, Sabloff $\mathrm{B}$, et al. Factors influencing pneumothorax rate at lung biopsy: are dwell time and angle of pleural puncture contributing factors? Radiology. 2001;218(2):491-6.

7. Iguchi $\mathrm{T}$, Hiraki $\mathrm{T}$, Ishii $\mathrm{H}$, et al. Transosseous route for $\mathrm{CT}$ fluoroscopy-guided radiofrequency ablation of lung tumors. J Vasc Interv Radiol. 2015;26(11):1694-8.

8. Rossi UG, Seitun S, Ferro C. MDCT-guided transthoracic needle aspiration biopsy of the lung using the trans-scapular approach. Cardiovasc Interv Radiol. 2011;34(1):184-7.

9. Patnaik VVG, Kalsey G, Singla RK. Branching pattern of axillary artery-a morphological study. J Anat Soc India. 2000;49:12732.

10. Grasso RF, Faiella E, Luppi G, et al. Percutaneous lung biopsy: comparison between an augmented reality $\mathrm{CT}$ navigation system and standard CT-guided technique. Int J Comput Assist Radiol Surg. 2013;8(5):837-48.

11. MacDuff A, Arnold A, Harvey J, BTS Pleural Disease Guideline Group. Management of spontaneous pneumothorax: British Thoracic Society pleural disease guideline 2010. Thorax. 2010;65(Suppl 2):ii18-31.

12. Sacks D, McClenny TE, Cardella JF, Lewis CA. Society of interventional radiology clinical practice guidelines. J Vasc Interv Radiol. 2003;14(9 Pt 2):S199-202. 
13. Basik M, Aguilar-Mahecha A, Rousseau C, et al. Biopsies: nextgeneration biospecimens for tailoring therapy. Nat Rev Clin Oncol. 2013;10:437-50.

14. Kim L, Tsao MS. Tumour tissue sampling for lung cancer management in the era of personalised therapy: what is good enough for molecular testing? Eur Respir J. 2014;44(4):1011-22.

15. Kauczor HU, Bonomo L, Gaga M, European Society of Radiology (ESR), European Respiratory Society (ERS), et al. ESR/ERS white paper on lung cancer screening. Eur Radiol. 2015;25(9):2519-31.

16. Chiari R, Metro G, Iacono D, et al. Clinical impact of sequential treatment with ALK-TKIs in patients with advanced ALK-positive non-small cell lung cancer: results of a multicenter analysis. Lung Cancer. 2015;90(2):255-60.

17. Ludovini V, Bianconi F, Siggillino A, Piobbico D, Vannucci J, Metro G, et al. Gene identification for risk of relapse in stage I lung adenocarcinoma patients: a combined methodology of gene expression profiling and computational gene network analysis. Oncotarget. 2016;7(21):30561-74.

18. Aktas AR, Gozlek E, Yazkan R, et al. Transthoracic biopsy of lung masses: nontechnical factors affecting complication occurrence. Thorac Cancer. 2015;6(2):151-8. doi:10.1111/1759-7714. 12156.

19. Yao X, Gomes MM, Tsao MS, Allen CJ, Geddie W, Sekhon H. Fine-needle aspiration biopsy versus core-needle biopsy in diagnosing lung cancer: a systematic review. Curr Oncol. 2012;19(1):e16-27.

20. Albanna AS, Kasymjanova G, Robitaille C, et al. Comparison of the yield of different diagnostic procedures for cellular differentiation and genetic profiling of non-small-cell lung cancer. J Thorac Oncol. 2014;9(8):1120-5. 


\title{
Erratum to: CT-Guided Percutaneous Trans-scapular Lung Biopsy in the Diagnosis of Peripheral Pulmonary Lesion Nodules of the Superior Lobes Using Large Needles
}

\author{
Alberto Rebonato $^{1}$ - Daniele Maiettini ${ }^{1}$ - Marco Andolfi ${ }^{2}$ - Matthias J. Fischer ${ }^{3}$. \\ Jacopo Vannucci ${ }^{2} \cdot$ Giulio Metro $^{4}$ - Antonio Basile ${ }^{5} \cdot$ Michele Duranti $^{3}$. \\ Michele Rossi ${ }^{6}$
}

(c) Springer Science+Business Media, LLC and the Cardiovascular and Interventional Radiological Society of Europe (CIRSE) 2017

\section{Erratum to: Cardiovasc Intervent Radiol DOI 10.1007/s00270-017-1768-0}

In the published article, Michele Rossi mistakenly appears as the next to the last name in the author list. Michele Rossi should appear as the last name in the author list as shown in this erratum.
The online version of the original article can be found under doi: $10.1007 / \mathrm{s} 00270-017-1768-0$.
Alberto Rebonato
rebonatoalberto@gmail.com
Daniele Maiettini
danielemaiettini@gmail.com
Marco Andolfi
marcoandolfi@hotmail.com
Matthias J. Fischer
mfischer@alice.it
Jacopo Vannucci
jacopovannucci@tiscali.it
Giulio Metro
giulio.metro@yahoo.com
Antonio Basile
antodoc@yahoo.com
Michele Duranti
micheleduranti@aopg.it
Michele Rossi
michele.rossi@uniroma1.it

Radiology Unit, Department of Surgical and Biomedical Science, Santa Maria della Misericordia University Hospital, Perugia University, Sant'Andrea delle Fratte, 06132 Perugia, Italy

2 Department of Thoracic Surgery, S. Maria della Misericordia Hospital, University of Perugia Medical School, Sant'Andrea delle Fratte, 06132 Perugia, Italy

3 Department of Radiology, S. Maria della Misericordia Hospital, University of Perugia Medical School, Sant'Andrea delle Fratte, 06132 Perugia, Italy

4 Department of Medical Oncology, S. Maria della Misericordia Hospital, University of Perugia Medical School, Sant'Andrea delle Fratte, 06132 Perugia, Italy

5 Department of Diagnostic and Interventional Radiology, Ospedale Ferrarotto, Via Trieste 14, 95127 Catania, Italy

6 Department of Radiology, Sant'Andrea Hospital, Faculty of Medicine and Psychology, Sapienza University, Rome, Italy 